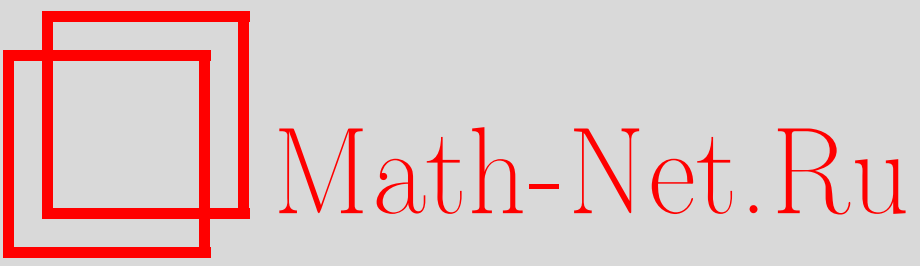

А. А. Татаркин, А. Б. Шишкин, Синтез в ядре оператора трехсторонней свертки, Итоги науки и техн. Сер. Соврем. мат. и ее прил. Темат. обз., 2021, том 193, 130-141

DOI: https://doi.org/10.36535/0233-6723-2021-193-130-141

Использование Общероссийского математического портала Math-Net.Ru подразумевает, что вы прочитали и согласны с пользовательским соглашением

http://www . mathnet.ru/rus/agreement

Параметры загрузки:

IP: 52.205 .19 .152

26 апреля 2023 г., 18:25:10 


\title{
СИНТЕЗ В ЯДРЕ ОПЕРАТОРА ТРЕХСТОРОННЕЙ СВЕРТКИ
}

\author{
(c) 2021 г. $\quad$ А. А. ТАТАРКИН, А. Б. ШИШКИН
}

\begin{abstract}
АннотАция. Говорят, что для однородного уравнения типа свертки справедлива аппроксимационная теорема, если любое решение этого уравнения аппроксимируется его элементарными решениями. В статье сформулировано необходимое и достаточное условие выполнимости аппроксимационной теоремы для однородного уравнения трехсторонней свертки при любом выборе выпуклой области и его характеристической функции.
\end{abstract}

Ключевые слова: экспоненциальный синтез, ядро оператора, оператор типа свертки, инвариантное подпространство, аналитическая функция.

\section{SYNTHESIS IN THE KERNEL OF THE THREE-WAY CONVOLUTION OPERATOR}

\author{
(c) 2021 A. A. TATARKIN, A. B. SHISHKIN
}

\begin{abstract}
One says that an approximation theorem holds for a homogeneous convolution-type equation if any solution of this equation is approximated by its elementary solutions. In this paper, we state a necessary and sufficient condition for the validity of the approximation theorem for the homogeneous equation of three-way convolution for any choice of a convex domain and its characteristic function.
\end{abstract}

Keywords and phrases: exponential synthesis, kernel of operator, convolution-type operator, invariant subspace, analytic function.

AMS Subject Classification: 34L05

1. Введение. Пусть $\Omega_{0}, \Omega$ - выпуклые области в комплексной плоскости $\mathbb{C}, \varepsilon>0, U_{\varepsilon}-$ круг $\{z:|z|<\varepsilon\}$. Будем считать, что $\Omega_{0}+U_{\varepsilon} \subseteq \Omega$ и пространства голоморфных функций $O\left(\Omega_{0}\right)$, $O\left(U_{\varepsilon}\right), O\left(\Omega_{0}\right)$ и $O(\mathbb{C})$ наделены топологиями равномерной сходимости на компактах. Выберем произвольный набор комплексных чисел $a_{0}, a_{1}, a_{2}$, не все из которых равны нулю. Символом $\omega_{3}$ обозначим комплексное число ехр $\frac{2 \pi i}{3}$. Пусть $A$-линейный непрерывный оператор, действующий в пространстве $O(\mathbb{C})$ по правилу

$$
g(\lambda) \rightarrow a_{0} g\left(\omega_{3}^{0} \lambda\right)+a_{1} g\left(\omega_{3}^{1} \lambda\right)+a_{2} g\left(\omega_{3}^{2} \lambda\right) ;
$$

$A T_{h}$ - линейный непрерывный оператор, действующий из пространства $O(\Omega)$ в пространство $O\left(\Omega_{0}\right)$ по правилу

$$
f(z) \rightarrow a_{0} f\left(z+\omega_{q}^{0} h\right)+a_{1} f\left(z+\omega_{q}^{1} h\right)+a_{2} f\left(z+\omega_{q}^{2} h\right) .
$$

Оператор $A T_{h}$ принято называть оператором трехстороннего сдвига (на шаг $h \in U_{\varepsilon}$ ).

Выберем произвольную функцию $f \in O(\Omega)$ и произвольный линейный непрерывный функционал $S$ на пространстве $O\left(\Omega_{0}\right)$. Функция

$$
\psi(h):=\left\langle S, T_{h}(f)\right\rangle
$$


называется сверткой функции $f$ и функционала $S$. При фиксированных $S$ и $\varepsilon$ оператор

$$
f \rightarrow \psi(h):=\left\langle S, T_{h}(f)\right\rangle
$$

называется оператором свертки. Известно, что он действует из пространства $O(\Omega)$ в пространство $O\left(U_{\varepsilon}\right)$ и является непрерывным (см. $\left.[2, \S 6]\right)$. Выберем произвольный оператор трехстороннего сдвига $A T_{h}: O(\Omega) \rightarrow O\left(\Omega_{0}\right)$, произвольную функцию $f \in O(\Omega)$ и произвольный линейный непрерывный функционал $S$ на пространстве $O\left(\Omega_{0}\right)$. Функция $\psi_{A}(h):=\left\langle S, A T_{h}(f)\right\rangle$ называется трехсторонней сверткой функции $f$ и функционала $S$. При фиксированных $S$ и $\varepsilon$ линейный оператор $f \rightarrow \psi_{A}(h):=\left\langle S, A T_{h}(f)\right\rangle$ называется оператором трехсторонней свертки. Легко убедиться, что оператор трехсторонней свертки действует из пространства $O(\Omega)$ в пространство $O\left(U_{\varepsilon}\right)$ и является непрерывным (см. $[4, \S 7])$. Он перестановочен с оператором трехкратного дифференцирования $D^{3}$, то есть в области $\Omega_{0}$ выполняется следующее соотношение

$$
D_{h}^{3}\left\langle S, A T_{h}(f)\right\rangle=\left\langle S, A T_{h}\left(D^{3} f\right)\right\rangle .
$$

Однородное уравнение типа свертки

$$
\left\langle S, A T_{h}(f)\right\rangle=0, \quad f \in O(\Omega),
$$

называется однородным уравнением трехсторонней свертки. Множество решений $f \in O(\Omega)$ однородного уравнения (1) обозначим символом $W_{S}$. Множество $W_{S}$ является замкнутым $D^{3}$-инвариантным подпространством в $O(\Omega)$, то есть справедлива импликация

$$
f \in W_{S} \Rightarrow D^{3} f \in W_{S} .
$$

Экспоненциальные полиномы, удовлетворяющие уравнению (1), принято называть элементарными решениями этого уравнения. Говорят, что для однородного уравнения (1) справедлива аппроксимационная теорема, если произвольное решение $f \in O(\Omega)$ этого уравнения можно аппроксимировать элементарными решениями в топологии пространства $O(\Omega)$.

Известно, что аппроксимационная теорема для однородного уравнения (1) справедлива, если лишь один из коэффициентов $a_{0}, a_{1}, a_{2}$ отличен от нуля. В этом случае однородное уравнение (1) равносильно однородному уравнению свертки

$$
\langle S, f(z+h)\rangle=0, \quad f \in O(\Omega) .
$$

Справедливость аппроксимационной теоремы для таких уравнений доказана в [2, теорема 6.1]. Кроме того, аппроксимационная теорема справедлива, если $a_{0}=a_{1}=a_{2}$. В этом случае однородное уравнение (1) равносильно однородному уравнению

$$
\left\langle S, f\left(z+\omega_{q}^{0} h\right)+f\left(z+\omega_{q}^{1} h\right)+f\left(z+\omega_{q}^{2} h\right)\right\rangle=0, \quad f \in O(\Omega) .
$$

Справедливость аппроксимационной теоремы для таких уравнений доказана в [4, теорема 7.1, следствие 7.3]. Возникает вопрос о справедливости аппроксимационной теоремы в случае произвольных коэффициентов $a_{0}, a_{1}, a_{2}$, произвольной выпуклой области $\Omega$ и произвольного $S$.

2. Формулировка основного результата. Оператор $A T_{h}$ является линейным и непрерывным. Он совпадает с дифференциальным оператором бесконечного порядка

$$
f(z) \rightarrow \sum_{n=0}^{\infty} b_{n} \frac{h^{n}}{n !}\left(D^{n} f\right)(z),
$$

где

$$
b_{n}:=a_{0}+a_{1} \omega_{q}^{n}+a_{2} \omega_{q}^{2 n}, \quad n \in\{0,1, \ldots\},
$$

и ряд (2) сходится равномерно на компактах из $\Omega_{0}$. Характеристическая функция оператора $A T_{h}$ совпадает с функцией

$$
A\left(e^{h \lambda}\right):=\sum_{n=0}^{\infty} b_{n} \frac{h^{n} \lambda^{n}}{n !} .
$$

Замечаем, что эти коэффициенты $b_{n}$ зависят от $n$ периодическим образом, то есть для любого $n \in \mathbb{Z}_{+}$выполняется равенство $b_{n+3}=b_{n}$. Значит, среди коэффициентов $b_{0}, b_{1}, b_{2}$ есть отличные 
от нуля. Отметим, что коэффициенты $a_{0}, a_{1}, a_{2}$ однозначно определяются по коэффициентам $b_{0}, b_{1}, b_{2}$ с помощью формулы $a_{k}=\Delta_{k} / \Delta$, где $\Delta_{k}$-определитель, полученный из определителя

$$
\Delta:=\left|\begin{array}{ccc}
1 & 1 & 1 \\
1 & \omega_{3}^{1} & \omega_{3}^{2} \\
1 & \omega_{3}^{2} & \omega_{3}^{4}
\end{array}\right|=\left(\omega_{3}-1\right)\left(\omega_{3}^{2}-1\right)\left(\omega_{3}^{2}-\omega_{3}\right) \neq 0,
$$

заменой $k$-го столбца на столбец, составленный из коэффициентов $b_{0}, b_{1}, b_{2}$.

Обозначим через $n_{A}:=\left\{n_{1}, \ldots, n_{\nu}\right\}$ упорядоченный набор целых неотрицательных чисел из множества $\{0,1,2\}$, удовлетворяющих следующим условиям:

(i) $0 \leqslant n_{1}<\ldots<n_{\nu} \leqslant 2$;

(ii) если $n \in\left\{n_{1}, \ldots, n_{\nu}\right\}$, то $b_{n} \neq 0$;

(iii) если $n \notin\left\{n_{1}, \ldots, n_{\nu}\right\}$, то $b_{n}=0$.

Упорядоченный набор целых чисел $n_{A}$, удовлетворяющий условиям (i)-(iii), будем называть индикатором уравнения (1). Дополним индикатор $n_{A}$ уравнения (1) одним элементом $n_{\nu+1}:=$ $n_{1}+3$. Будем говорить, что индикатор $n_{A}$ периодичен, если существует такое $q \in \mathbb{N}$, что для всех $k \in\{1, \ldots, \nu\}$ выполняется равенство

$$
n_{k+1}=n_{k}+q
$$

Отметим, что индикатор $n_{A}$ периодичен, если $\nu=1$ или $\nu=3$. Действительно, если $\nu=1$, то индикатор $n_{A}$ включает лишь один элемент $n_{1}$ и условие периодичности содержит лишь одно равенство $n_{2}=n_{1}+q$, в котором можно положить $q:=3$. В случае $\nu=3$ индикатор $n_{A}$ включает три элемента $n_{1}:=0, n_{2}=1$ и $n_{3}=2$, значит, условие периодичности содержит три равенства $n_{2}=n_{1}+q, n_{3}=n_{2}+q$ и $n_{4}=n_{3}+q$, в которых можно положить $q:=1$.

Справедлива следующая теорема.

Теорема 1. Аппроксимачионная теорема для однородного уравнения трехсторонней свертки (1) справедлива при любом выборе выпуклой области $\Omega$ и функиионала $S$ тогда и только тогда, когда индикатор $n_{A}$ этого уравнения периодичен.

3. Спектральный синтез. Пусть $O^{*}(\Omega)$ - сильное сопряженное к пространству $O(\Omega) ; L_{\Omega}-$ преобразование Лапласа, которое каждому функционалу $S \in O^{*}(\Omega)$ ставит в соответствие целую функцию экспоненциального типа $\varphi(\lambda):=\left\langle S, e^{\lambda z}\right\rangle ; P(\Omega)$ - полный образ $L_{\Omega}$. Известно, что отображение $L_{\Omega}: O^{*}(\Omega) \rightarrow P(\Omega)$ является взаимно однозначным. Оно индуцирует в $P(\Omega)$ отделимую локально выпуклую топологию. Известно, что пространство $P(\Omega)$ совпадает с индуктивным пределом $P\left[1, H_{\Omega}\right)$, где $H_{\Omega}$ - опорная функция области $\Omega$ в смысле комплексного анализа (см. [1, предложение 3.1]). Так как $L_{\Omega}$ осуществляет изоморфизм локально выпуклых пространств, то его сопряженное отображение $L_{\Omega}^{*}$ осуществляет изоморфизм сильного сопряженного пространства $P^{*}(\Omega)$ на второе сопряженное пространство $O^{* *} \Omega$. Воспользуемся рефлексивностью пространства $O(\Omega)$ и отождествим это пространство с его вторым сопряженным пространством.

Соблюдая традицию, оператор умножения $O(\mathbb{C}) \rightarrow O(\mathbb{C})$ на одночлен $\pi(z):=z^{3}$ обозначим $\pi$, а его сопряженный оператор обозначим $\pi^{*}$. Символом $\Delta_{\lambda}$ обозначим корневое подпространство оператора $\pi^{*}$, соответствующее собственному значению $\lambda$. Известно, что алгебраический спектр оператора $\pi^{*}$ совпадает с $\mathbb{C}$, а корневое подпространство $\Delta_{\lambda} \subseteq O^{*}(\mathbb{C})$ совпадает с линейной оболочкой множества

$$
\left\{\delta_{\xi}^{(j)}:(j, \xi) \in \mathbb{Z}_{+} \times \lambda^{1 / 3}\right\},
$$

где $\delta_{\xi}^{(j)} \in O^{*}(\mathbb{C})$ - функционал, действующий по правилу $f \rightarrow f^{(j)}(\xi)$ (см. [4, теорема 1.1]).

Пространство $P(\Omega)$ является всюду плотным подпространством $O(\mathbb{C})$ и вложение $P(\Omega) \subseteq O(\mathbb{C})$ является непрерывным. Значит, сильное сопряженное пространство $O^{*}(\mathbb{C})$ можно отождествить с подпространством сильного сопряженного $P^{*}(\Omega)$. При этом вложение $O^{*}(\mathbb{C}) \subseteq P^{*}(\Omega)$ является непрерывным. Говорят, что корневой элемент $s \in \Delta_{\lambda}$ погружен в подпространство $V \subseteq P^{*}(\Omega)$, если $\left(\pi^{*}-\lambda\right)^{j} s \in V$ для любого $j \in \mathbb{Z}_{+}$. Замкнутое подпространство $V \subseteq P^{*}(\Omega)$ допускает спектральный синтез (синтез по корневым элементам оператора $\pi^{*}$ ), если оно совпадает с замыканием линейной оболочки множества корневых элементов оператора $\pi^{*}$, погруженных в $V$. 
Рассмотрим дуальный оператор $\pi^{\circledast}$ к оператору $\pi: O(\mathbb{C}) \rightarrow O(\mathbb{C})$ (см. [6, §3]). Согласно определению оператор $\pi^{\circledast}$ действует из пространства $P(\mathbb{C})$ в пространство $P(\mathbb{C})$ и совпадает с композицией $L_{\mathbb{C}} \circ \pi^{*} \circ L_{\mathbb{C}}^{-1}$. В $[6, \S 6$, п. 2] доказаны следующие утверждения:

(а) алгебраический спектр оператора $\pi^{\circledast}$ совпадает с $\mathbb{C}$, а корневое подпространство $E_{\lambda} \subseteq P(\mathbb{C})$ оператора $\pi^{\circledast}$, соответствующее собственному значению $\lambda$, совпадает с линейной оболочкой множества экспоненциальных одночленов

$$
\left\{z^{j} e^{\xi z}:(j, \xi) \in \mathbb{Z}_{+} \times \lambda^{1 / 3}\right\}
$$

(b) замкнутое подпространство $W_{S} \subseteq O(\Omega)$ допускает синтез по корневым элементам оператора $\pi^{\circledast}$ тогда и только тогда, когда его дуальное подпространство $V_{S}:=\left(L_{\Omega}^{*}\right)^{-1}\left(W_{S}\right) \subseteq P^{*}(\Omega)$ допускает синтез по корневым элементам оператора $\pi^{*}$.

Из (a) и (b) вытекает справедливость следующего предложения.

Предложение 1. Аппроксимачионная теорема для однородного уравнения трехсторонней свертки (1) справедлива тогда и только тогда, когда дуальное подпространство $V_{S} \subseteq P^{*}(\Omega)$ допускает синтез по корневым элементам оператора $\pi^{*}$.

Доказательство. Из (а) вытекает, что аппроксимационная теорема для однородного уравнения трехсторонней свертки (1) справедлива тогда и только тогда, когда замкнутое подпространство $W_{S} \subseteq O(\Omega)$ допускает синтез по корневым элементам оператора $\pi^{\circledast}$. Осталось сослаться на условие (b). Предложение доказано.

4. Плотность многочленов. Пусть $n_{A}:=\left\{n_{1}, \ldots, n_{\nu}\right\}$ - индикатор уравнения (1). Векторную функцию $\boldsymbol{g}:=\left(g_{1}, \ldots, g_{\nu}\right)$ называем целой $\nu$-функцией, если все функции $g_{1}, \ldots, g_{\nu}$ являются целыми. Если целые функции $g_{1}, \ldots, g_{\nu}$ являются полиномами, то целую $\nu$-функцию $\boldsymbol{g}:=\left(g_{1}, \ldots, g_{\nu}\right)$ называем $\nu$-полиномом.

Выберем произвольную функцию $\varphi \in P(\Omega)$ и обозначим $\boldsymbol{O}_{\varphi}$ пространство всех целых $\nu$-функций $\boldsymbol{g}:=\left(g_{1}, \ldots, g_{\nu}\right)$, для которых сумма

$$
z^{n_{1}} g_{1}\left(z^{q}\right) \varphi(z)+\ldots+z^{n_{\nu}} g_{\nu}\left(z^{q}\right) \varphi(z)
$$

принадлежит $P(\Omega)$. Определим отображение $\boldsymbol{u}_{\varphi}: \boldsymbol{O}_{\varphi} \rightarrow P(\Omega)$ по правилу

$$
\boldsymbol{g} \rightarrow z^{n_{1}} g_{1}\left(z^{q}\right) \varphi(z)+\ldots+z^{n_{\nu}} g_{\nu}\left(z^{q}\right) \varphi(z) .
$$

Символом $\Delta_{n_{A}}$ обозначим определитель Вандермонда

$$
\left|\begin{array}{ccc}
\omega_{q}^{0} & \ldots & \omega_{q}^{0} \\
\omega_{q}^{n_{1}} & \ldots & \omega_{q}^{n_{\nu}} \\
\ldots \ldots \ldots \ldots \ldots & \ldots \ldots \ldots \\
\omega_{q}^{(\nu-1) n_{1}} & \ldots & \omega_{q}^{(\nu-1) n_{\nu}}
\end{array}\right|
$$

По известной формуле имеем

$$
\Delta_{n_{A}}:=\prod_{1 \leqslant i<j \leqslant \nu}\left(\omega_{q}^{n_{i}}-\omega_{q}^{n_{j}}\right) \neq 0 .
$$

Для произвольной целой функций $\psi$ символом

$$
\Delta_{n_{A}}^{k}\left(\psi(z), \psi\left(\omega_{q} z\right), \ldots, \psi\left(\omega_{q}^{\nu-1} z\right)\right)
$$

обозначим определитель

$$
\left|\begin{array}{ccccc}
\omega_{q}^{0} & \ldots & \psi(z) & \ldots & \omega_{q}^{0} \\
\omega_{q}^{n_{1}} & \ldots & \psi\left(\omega_{q} z\right) & \ldots & \omega_{q}^{n_{\nu}} \\
\ldots \ldots \ldots \ldots \ldots \ldots & \ldots \ldots \ldots \ldots \ldots \ldots \ldots \ldots \ldots \\
\omega_{q}^{(\nu-1) n_{1}} & \ldots & \psi\left(\omega_{q}^{\nu-1} z\right) & \ldots & \omega_{q}^{(\nu-1) n_{\nu}}
\end{array}\right|
$$

который получен из определителя Вандермонда $\Delta_{n_{A}}$ заменой $k$-го столбца на столбец из функций $\psi(z), \psi\left(\omega_{q} z\right), \ldots, \psi\left(\omega_{q}^{\nu-1} z\right)$. 
Предложение 2. Справедливы следующие утверждения:

(i) отображение $\boldsymbol{u}_{\varphi}: \boldsymbol{O}_{\varphi} \rightarrow P(\Omega)$ является взаимно однозначным;

(ii) полный образ $\boldsymbol{u}_{\varphi}\left(\boldsymbol{O}_{\varphi}\right)$ исчерпывается произведениями $\psi \varphi \in P(\Omega)$, где $\psi$-такая целая функиия, что для любого $k \in\{1, \ldots, \nu\}$ функиия

$$
\frac{\Delta_{n_{A}}^{k}\left(\psi(z), \psi\left(\omega_{q} z\right), \ldots, \psi\left(\omega_{q}^{\nu-1} z\right)\right)}{z^{n_{k}} \Delta_{n_{A}}}
$$

представляется в виде композичии $g_{k}\left(z^{q}\right)$, где $g_{k}$ - целая функиия, то есть является целой q-симметричной функиией;

(iii) Полный образ $\boldsymbol{u}_{\varphi}\left(\boldsymbol{O}_{\varphi}\right)$ замкнут в пространстве $O(\mathbb{C})$.

Доказательство. Докажем утверждение (i). Пусть $\boldsymbol{g}^{(1)}:=\left(g_{1}^{(1)}, \ldots, g_{\nu}^{(1)}\right)$ и $\boldsymbol{g}^{(2)}:=\left(g_{1}^{(2)}, \ldots, g_{\nu}^{(2)}\right)$ принадлежат $\boldsymbol{O}_{\varphi}$ и $\boldsymbol{u}_{\varphi}\left(\boldsymbol{g}^{(1)}\right)=\boldsymbol{u}_{\varphi}\left(\boldsymbol{g}^{(2)}\right)$. Тогда вне множества нулей целой функции $\varphi$ выполняются равенства:

$$
\begin{gathered}
z^{n_{1}} g_{1}^{(1)}\left(z^{q}\right)+\ldots+z^{n_{\nu}} g_{\nu}^{(1)}\left(z^{q}\right)=z^{n_{1}} g_{1}^{(2)}\left(z^{q}\right)+\ldots+z^{n_{\nu}} g_{\nu}^{(2)}\left(z^{q}\right), \\
z^{n_{1}}\left(g_{1}^{(1)}\left(z^{q}\right)-g_{1}^{(2)}\left(z^{q}\right)\right)+\ldots+z^{n_{\nu}}\left(g_{\nu}^{(1)}\left(z^{q}\right)-g_{\nu}^{(2)}\left(z^{q}\right)\right)=0 .
\end{gathered}
$$

При этом для целых функций

$$
g_{1}(z):=g_{1}^{(1)}\left(z^{q}\right)-g_{1}^{(2)}\left(z^{q}\right), \ldots, g_{\nu}(z):=g_{\nu}^{(1)}\left(z^{q}\right)-g_{\nu}^{(2)}\left(z^{q}\right)
$$

справедливы соотношения

$$
g_{1}\left(\omega_{q}^{k} z\right)=g_{1}(z), \ldots, g_{1}\left(\omega_{q}^{k} z\right)=g_{\nu}(z), \quad k \in \mathbb{N},
$$

где $\omega_{q}:=\exp \frac{2 \pi i}{q}$. Отсюда вытекает, что функции

$$
z^{n_{1}} g_{1}(z), \ldots, z^{n_{\nu}} g_{\nu}(z)
$$

вне множества нулей функции $\varphi$ удовлетворяют системе уравнений

$$
\left\{\begin{array}{l}
z^{n_{1}} g_{1}(z)+\ldots+z^{n_{\nu}} g_{\nu}(z)=0 \\
\omega_{q}^{n_{1}} z^{n_{1}} g_{1}(z)+\ldots+\omega_{q}^{n_{\nu}} z^{n_{\nu}} g_{\nu}(z)=0 \\
\ldots \ldots \ldots \ldots \ldots \ldots \ldots \ldots \ldots \\
\omega_{q}^{(\nu-1) n_{1}} z^{n_{1}} g_{1}(z)+\ldots+\omega_{q}^{(\nu-1) n_{\nu}} z^{n_{\nu}} g_{\nu}(z)=0
\end{array}\right.
$$

Определитель этой системы совпадает с определителем Вандермонда

$$
\Delta_{n_{A}}:=\left|\begin{array}{ccc}
\omega_{q}^{0} & \ldots & \omega_{q}^{0} \\
\omega_{q}^{n_{1}} & \ldots & \omega_{q}^{n_{\nu}} \\
\ldots \ldots \ldots \ldots & \ldots & \ldots \ldots \ldots \\
\ldots(\nu-1) n_{1} & \ldots & \omega_{q}^{(\nu-1) n_{\nu}}
\end{array}\right|=\prod_{1 \leqslant i<j \leqslant \nu}\left(\omega_{q}^{n_{i}}-\omega_{q}^{n_{j}}\right) .
$$

Это значит, что определитель указанной системы отличен от нуля. Следовательно, функции $z^{n_{1}} g_{1}(z), \ldots, z^{n_{\nu}} g_{\nu}(z)$ равны нулю вне нулей функции $\varphi$. Отсюда следует, что целые функции $g_{1}(z), \ldots, g_{\nu}(z)$ являются тождественными нулями. Значит, $\boldsymbol{g}^{(1)}=\boldsymbol{g}^{(2)}$. Таким образом, взаимная однозначность отображения $\boldsymbol{u}_{\varphi}: \boldsymbol{O}_{\varphi} \rightarrow P(\Omega)$ доказана.

Докажем утверждение (ii). Если $\boldsymbol{g}:=\left(g_{1}, \ldots, g_{\nu}\right) \in \boldsymbol{O}_{\varphi}$, то $f:=\boldsymbol{u}_{\varphi}(\boldsymbol{g}) \in P(\Omega)$. При этом для любого $z \in \mathbb{C}$ имеем

$$
\begin{aligned}
f(z) & =z^{n_{1}} g_{1}\left(z^{q}\right) \varphi(z)+\ldots+z^{n_{\nu}} g_{\nu}\left(z^{q}\right) \varphi(z)= \\
& =\left(z^{n_{1}} g_{1}\left(z^{q}\right)+\ldots+z^{n_{\nu}} g_{\nu}\left(z^{q}\right)\right) \varphi(z)
\end{aligned}
$$

или

$$
z^{n_{1}} g_{1}\left(z^{q}\right)+\ldots+z^{n_{\nu}} g_{\nu}\left(z^{q}\right)=\psi(z)
$$


где $\psi$ - целая функция $\frac{f}{\varphi}$. Значит, для всех комплексных $z$ выполняются равенства

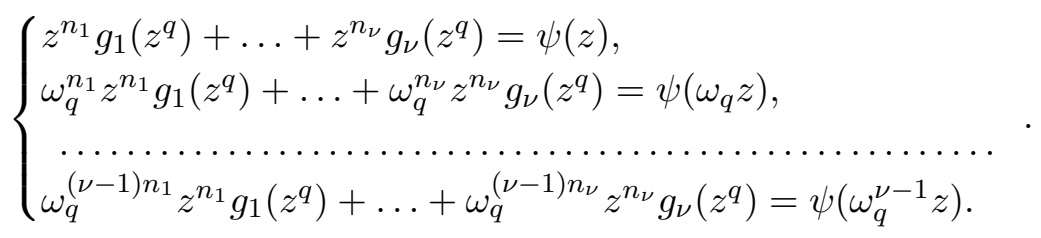

Следовательно, для произвольного $k \in\{1, \ldots, \nu\}$ имеем

$$
g_{k}\left(z^{q}\right)=\frac{\Delta_{n_{A}}^{k}\left(\psi(z), \psi\left(\omega_{q} z\right), \ldots, \psi\left(\omega_{q}^{\nu-1} z\right)\right)}{z^{n_{k}} \Delta_{n_{A}}} .
$$

При этом $g_{1}, \ldots, g_{\nu}$ - целые функции и $f=\psi \varphi$.

Обратно, пусть $f=\psi \varphi \in P(\Omega)$, где целая функция $\psi$ выбрана из условия: для любого $k \in$ $\{1, \ldots, \nu\}$ функция

$$
\frac{\Delta_{n_{A}}^{k}\left(\psi(z), \psi\left(\omega_{q} z\right), \ldots, \psi\left(\omega_{q}^{\nu-1} z\right)\right)}{z^{n_{k}} \Delta_{n_{A}}}
$$

представляется в виде композиции $g_{k}\left(z^{q}\right)$, где $g_{k}$-целая функция. Тогда для целой $\nu$-функции $\boldsymbol{g}=\left(g_{1}, \ldots, g_{\nu}\right)$ имеем

$$
\begin{aligned}
\boldsymbol{u}_{\varphi}(\boldsymbol{g})=z^{n_{1}} g_{1}\left(z^{q}\right) \varphi(z)+\ldots+z^{n_{\nu}} g_{\nu}\left(z^{q}\right) \varphi(z) & = \\
& =\varphi(z) \sum_{k=1}^{\nu} \frac{\Delta_{n_{A}}^{k}\left(\psi(z), \psi\left(\omega_{q} z\right), \ldots, \psi\left(\omega_{q}^{\nu-1} z\right)\right)}{\Delta_{n_{A}}}=\varphi(z) \psi(z)=f(z) .
\end{aligned}
$$

Значит, утверждение (ii) справедливо.

Докажем утверждение (iii). Пусть $f \in O(\mathbb{C}), f_{m} \in \boldsymbol{u}_{\varphi}\left(\boldsymbol{O}_{\varphi}\right)$ и $f_{m} \rightarrow f$ в пространстве $O(\mathbb{C})$. Нужно доказать, что $f \in \boldsymbol{u}_{\varphi}\left(\boldsymbol{O}_{\varphi}\right)$. По определению отображения $\boldsymbol{u}_{\varphi}: \boldsymbol{O}_{\varphi} \rightarrow P(\Omega)$ элементы последовательности $f_{m}$ допускают представление

$$
f_{m}(z):=\boldsymbol{u}_{\varphi}\left(\boldsymbol{g}^{(m)}\right)=z^{n_{1}} g_{1}^{(m)}\left(z^{q}\right) \varphi(z)+\ldots+z^{n_{\nu}} g_{\nu}^{(m)}\left(z^{q}\right) \varphi(z)=\psi_{m}(z) \varphi(z),
$$

где

$$
\psi_{m}(z):=z^{n_{1}} g_{1}^{(m)}\left(z^{q}\right)+\ldots+z^{n_{\nu}} g_{\nu}^{(m)}\left(z^{q}\right), \quad \boldsymbol{g}^{(m)}:=\left(g_{1}^{(m)}, \ldots, g_{\nu}^{(m)}\right)
$$

- некоторые целые $\nu$-функции. Значит, предельная функция $f$ делится на функцию $\varphi$ и

$$
\psi_{m} \rightarrow \psi:=\frac{f}{\varphi}
$$

в пространстве $O(\mathbb{C})$. Согласно утверждению (ii) для любого $k \in\{1, \ldots, \nu\}$ элементы последовательности $g_{k}^{(m)}\left(z^{q}\right)$ допускают представление

$$
g_{k}^{(m)}\left(z^{q}\right)=\frac{\Delta_{n_{A}}^{k}\left(\psi_{m}(z), \psi_{m}\left(\omega_{q} z\right), \ldots, \psi_{m}\left(\omega_{q}^{\nu-1} z\right)\right)}{z^{n_{k}} \Delta_{n_{A}}}
$$

Значит,

$$
g_{k}^{(m)}\left(z^{q}\right) \rightarrow g_{k}\left(z^{q}\right):=\frac{\Delta_{n_{A}}^{k}\left(\psi(z), \psi\left(\omega_{q} z\right), \ldots, \psi\left(\omega_{q}^{\nu-1} z\right)\right)}{z^{n_{k}} \Delta_{n_{A}}}
$$

в пространстве $O(\mathbb{C})$. При этом на всей комплексной плоскости имеет место представление

$$
\psi(z):=z^{n_{1}} g_{1}\left(z^{q}\right) \varphi(z)+\ldots+z^{n_{\nu}} g_{\nu}\left(z^{q}\right) \varphi(z) .
$$

Это и означает, что множество $\boldsymbol{u}_{\varphi}\left(\boldsymbol{O}_{\varphi}\right)$ замкнуто в пространстве $O(\mathbb{C})$.

По предложению 2 отображение $\boldsymbol{u}_{\varphi}: \boldsymbol{O}_{\varphi} \rightarrow P(\Omega)$ является взаимно однозначным. Наделим $\boldsymbol{O}_{\varphi}$ локально выпуклой топологией, индуцированной из пространства $P(\Omega)$ отображением $\boldsymbol{u}_{\varphi}$. Так как $\varphi \in P(\Omega)$ и оператор умножения на многочлен является эндоморфизмом пространства $P(\Omega)$, то пространство $\boldsymbol{O}_{\varphi}$ включает в себя все $\nu$-полиномы. Принципиальное значение имеет вопрос плотности множества всех $\nu$-полиномов в пространстве $\boldsymbol{O}_{\varphi}$. Действительно, справедлива следующая теорема. 
Теорема 2. Следующие утверждения эквивалентны:

(i) дуальное подпространство $V_{S} \subseteq P^{*}(\Omega)$ допускает синтез по корневым элементам операmора $\pi^{*}$,

(ii) $\nu$-полиномы плотны в пространстве $\boldsymbol{O}_{\varphi}$.

Доказательство. Согласно [5, теорема 3] вытекает, что утверждение (i) выполняется тогда и только тогда, когда дуальное подпространство $V_{S} \subseteq P^{*}(\Omega)$ допускает инъективное описание (см. [5, п. 1.3]). С другой стороны, по теореме 4 из той же работы допустимость инъективного описания подпространством $V_{S}$ равносильна плотности $\nu$-полиномов в пространстве $\boldsymbol{O}_{\varphi}$. Теорема доказана.

В силу предложения 1 теорема 2 сводит доказательство аппроксимационной теоремы для однородного уравнения трехсторонней свертки (1) к доказательству плотности $\nu$-полиномов в пространстве $\boldsymbol{O}_{\varphi}$.

5. Независимое описание топологии пространства $\boldsymbol{O}_{\varphi}$. Предположим, что область $\Omega$ является выпуклой, ограниченной и содержит начало. Так как $0 \in \Omega$, то при некотором $\rho>0$ круг $U_{\rho}:=\{z:|z|<\rho\}$ лежит в области $\Omega$. Пусть

$$
h_{\Omega}(\theta):=\sup _{z \in \Omega} \operatorname{Re}(z \exp \{-i \theta\})
$$

- опорная функция области $\Omega$ в смысле комплексного анализа. Для любого $\theta \in \mathbb{R}$ выполняется очевидное неравенство $h_{\Omega}(\theta)>\rho$. Выберем натуральное $m$ из условия $m>\rho^{-1}$ и символом $\boldsymbol{O}_{\varphi, m}$ обозначим векторное пространство всех целых $\nu$-функций $\boldsymbol{g}:=\left(g_{1}, \ldots, g_{\nu}\right)$, для которых

$$
\sup _{z \in \mathbb{C}} \frac{\left|z^{n_{1}} g_{1}\left(z^{q}\right) \varphi(z)+\ldots+z^{n_{\nu}} g_{\nu}\left(z^{q}\right) \varphi(z)\right|}{\exp h_{m}(z)}<+\infty,
$$

где $n_{A}:=\left\{n_{1}, \ldots, n_{\nu}\right\}$ - индикатор оператора $q$-стороннего сдвига $A T_{h}$ и $h_{m}$ - неотрицательная функция

$$
z \rightarrow\left(h_{\Omega}(\arg z)-\frac{1}{m}\right)|z| .
$$

Используя предложение 2, легко убедиться, что соотношение

$$
\|\boldsymbol{g}\|_{m}:=\sup _{z \in \mathbb{C}} \frac{\left|z^{n_{1}} g_{1}\left(z^{q}\right) \varphi(z)+\ldots+z^{n_{\nu}} g_{\nu}\left(z^{q}\right) \varphi(z)\right|}{\exp h_{m}(z)}
$$

определяет норму на пространстве $\boldsymbol{O}_{\varphi, m}$. При этом пространство $\boldsymbol{O}_{\varphi, m}$ являются банаховым, и вложение $\boldsymbol{O}_{\varphi, m} \subseteq \boldsymbol{O}_{\varphi, m+1}$ является вполне непрерывным. Хорошо известное описание топологии пространства $P(\Omega)$ (см. [1, предложение 3.1]) позволяет заключить, что пространство $\boldsymbol{O}_{\varphi}$ совпадает с индуктивным пределом пространств $\boldsymbol{O}_{\varphi, m}:=\left(\boldsymbol{O}_{\varphi, m} ;\|g\|_{m}\right)$ относительно вполне непрерывных вложений $\boldsymbol{O}_{\varphi, m} \subseteq \boldsymbol{O}_{\varphi, m+1}, m>\rho^{-1}$.

\section{6. Доказательство (аппроксимационной) теоремы 1.}

Достаточность. Предположим, что индикатор $n_{A}:=\left\{n_{1}, \ldots, n_{\nu}\right\}$ уравнения (1) периодичен, т.е.

$$
n_{2}-n_{1}=n_{3}-n_{2}=\ldots=n_{\nu+1}-n_{\nu}
$$

где $n_{\nu+1}:=n_{1}+3$. Это означает, что выполняется одно из условий: $n_{A}:=\left\{n_{1}\right\}$, то есть индикатор состоит из одного элемента; $n_{A}:=\{0,1,2\}$, то есть индикатор состоит из трех элементов.

В первом случае аппроксимационная теорема для однородного уравнения трехсторонней свертки (1) справедлива так как уравнение (1) в этом случае равносильно однородному уравнению

$$
\left\langle S, f\left(z+\omega_{q}^{0} h\right)+f\left(z+\omega_{q}^{1} h\right)+f\left(z+\omega_{q}^{2} h\right)\right\rangle=0 .
$$

Действительно, система линейных уравнений

$$
a_{0}+\omega_{q}^{n} a_{1}+\omega_{q}^{2 n} a_{2}=b_{n}, \quad n \in\{0,1,2\}
$$

в рассматриваемом случае имеет единственное решение $a_{0}=a_{1}=a_{2}=b_{0} / 3$. 
Рассмотрим второй случай. После кратного дифференцирования функции $\psi(h):=\left\langle S, A T_{h}(f)\right\rangle$ по $h$ получаем

$$
\begin{aligned}
\psi^{(n)}(h):= & \left\langle S, a_{0} f^{(n)}\left(z+\omega_{q}^{0} h\right)+\omega_{q}^{n} a_{1} f^{(n)}\left(z+\omega_{q}^{1} h\right)+\omega_{q}^{2 n} a_{2} f^{(n)}\left(z+\omega_{q}^{2} h\right)\right\rangle, \\
& \psi^{(n)}(0):=\left\langle S,\left(a_{0}+\omega_{q}^{n} a_{1}+\omega_{q}^{2 n} a_{2}\right) f^{(n)}\right\rangle=b_{n}\left\langle S, f^{(n)}\right\rangle .
\end{aligned}
$$

Если $f$ является решением однородного уравнения трехсторонней свертки $(1)$, то $\psi^{(n)}(h)=0$ для любого $h$ из окрестности нуля, значит, $b_{n}\left\langle S, f^{(n)}\right\rangle=0$ для любого $n \in\{0,1, \ldots\}$. Так как во втором случае все коэффициенты $b_{n}$ отличны от нуля, то $\left\langle S, f^{(n)}\right\rangle=0$ для любого $n \in\{0,1, \ldots\}$ Это означает, что функция $f$ удовлетворяет уравнению $\left\langle S, T_{h}(f)\right\rangle=0$. Следовательно, однородное уравнение трехсторонней свертки (1) во втором случае равносильно однородному уравнению свертки $\left\langle S, T_{h}(f)\right\rangle=0$.

Необходимость. Рассмотрим произвольное однородное уравнение трехсторонней свертки

$$
\left\langle S, \sum_{n=0}^{2} a_{n} f\left(z+\omega_{3}^{n} h\right)\right\rangle=0, \quad f \in O(\Omega),
$$

с индикатором $n_{A}:=\left\{n_{1}, n_{2}\right\}$. Предположим, что аппроксимационная теорема для однородного уравнения трехсторонней свертки (3) справедлива при любом выборе выпуклой области $\Omega$ и функционала $S$. Дополним индикатор $n_{A}$ двумя элементами $n_{3}:=n_{1}+3$ и $n_{4}:=n_{2}+3$. Замечаем, что $0<n_{3}-n_{2}=3-\left(n_{2}-n_{1}\right)<3$ и $0<n_{4}-n_{3}=n_{2}-n_{1}<3$, значит,

$$
0<n_{k+1}-n_{k}<3
$$

при любом $k \in\{1,2,3\}$. Условие периодичности индикатора $n_{A}:=\left\{n_{1}, n_{2}\right\}$ в рассматриваемом случае равносильно условию

$$
n_{2}-n_{1}=n_{3}-n_{2}=n_{4}-n_{3} .
$$

Допустим, что это условие нарушено. Тогда существует такое $s \in\{1,2\}$, что $n_{s+1}-n_{s}<n_{s+2}-n_{s+1}$ или

$$
2 n_{s+1}-n_{s}<n_{s+2} .
$$

Выберем произвольное число $\theta \in(0, \pi / 3)$ и обозначим символом $G_{0}$ угловую область $\{z$ : $|\arg z|<\theta\}$, а символом $G_{0}^{\prime}$ обозначим угловую область $\{z:|\arg z-\pi / 3|<\pi / 3-\theta\}$. Пусть

$$
\begin{gathered}
G_{1}:=\omega_{3}^{1} G_{0}, \quad G_{2}:=\omega_{3}^{2} G_{0}, \quad G_{1}^{\prime}:=\omega_{3}^{1} G_{0}^{\prime}, \quad G_{2}^{\prime}:=\omega_{3}^{2} G_{0}^{\prime}, \\
G=G_{0} \cup G_{1} \cup G_{2}, \quad G^{\prime}=G_{0}^{\prime} \cup G_{1}^{\prime} \cup G_{2}^{\prime} .
\end{gathered}
$$

Согласно свойствам квазиполиномов (см. [3, теорема 1.2.10]) нули $z_{m}^{(k)}$ целой 3-симметричной функции

$$
e\left(z^{3}\right):=\sum_{k=0}^{2} \exp \omega_{3}^{k} z
$$

имеют вид

$$
z_{m}^{(k)}=\frac{2 \pi m i}{\omega_{3}^{k+1}-\omega_{3}^{k}}+c_{k}+\delta_{m}^{(k)}, \quad c_{k} \in \mathbb{C}, \quad k \in\{0,1,2\},
$$

где $\left|\delta_{m}^{(k)}\right|<e^{-\varepsilon_{0} m}$ при некотором $\varepsilon_{0}>0$ и всех достаточно больших $m$. Лучи

$$
z=\frac{2 \pi i}{\omega_{3}^{k+1}-\omega_{3}^{k}} t, \quad k \in\{0,1,2\}
$$

являются серединными для угловых областей $G_{0}^{\prime}, G_{1}^{\prime}, G_{2}^{\prime}$ и при этом $G^{\prime}=\mathbb{C} \backslash \bar{G}$. Значит, лишь конечное число нулей 3 -симметричного квазиполинома $e\left(z^{3}\right)$ лежит в замыкании $\bar{G}$ множества $G$. 
Следовательно, при надлежащем выборе 3-симметричного полинома $p\left(z^{3}\right)$ все нули целой 3-симметричной функции

$$
A\left(z^{3}\right):=\frac{e\left(z^{3}\right)}{p\left(z^{3}\right)}=\frac{1}{p\left(z^{3}\right)} \sum_{k=0}^{2} \exp \omega_{3}^{k} z
$$

будут лежать в множестве $G^{\prime}=\mathbb{C} \backslash \bar{G}$. При этом все нули целой 3 -симметричной функции

$$
B\left(z^{3}\right):=\frac{1}{p\left(-z^{3}\right)} \sum_{k=0}^{2} \exp \omega_{6}^{2 k+1} z,
$$

где $\omega_{6}:=\exp \frac{\pi}{3} i$, будут лежать в множестве $G$. Сопряженные диаграммы $\bar{D}_{A}, \bar{D}_{B}$ функций $A\left(z^{3}\right)$ и $B\left(z^{3}\right)$ совпадают с правильными 3 -угольниками с вершинами в точках

$$
z_{0, A}=\omega_{6}^{0}=\omega_{3}^{0}, \quad z_{1, A}=\omega_{6}^{2}=\omega_{3}^{1}, \quad z_{2, A}=\omega_{6}^{4}=\omega_{3}^{2}
$$

и в точках

$$
z_{0, B}=\omega_{6}^{1}, \quad z_{1, B}=\omega_{6}^{3}, \quad z_{2, B}=\omega_{6}^{5}
$$

соответственно. Кроме того, $\bar{D}_{B}=\omega_{6} \bar{D}_{A}$. Пусть $\varsigma$ - вершина правильного шестиугольника $\bar{D}_{A} \cap$ $\bar{D}_{B}$, лежащая на пересечении двух прямых: первая из этих прямых проходит через точки $z_{0, A}:=$ $1, z_{1, A}:=\omega_{3}$ и задается уравнением $\operatorname{Re} \omega_{6}^{-1} z=\cos \frac{\pi}{3}$, а вторая прямая проходит через точки $z_{0, B}:=\omega_{6}, z_{2, B}:=\omega_{6}^{5}=\bar{\omega}_{6}$ и задается уравнением $\operatorname{Re} z=\cos \frac{\pi}{3}$. Решая систему из этих уравнений, получаем

$$
\varsigma=\cos \frac{\pi}{3}+i \operatorname{tg} \frac{\pi}{6} \cos \frac{\pi}{3}=\frac{1}{2}+i \frac{1}{2 \sqrt{3}} .
$$

Выберем $r \in\left(r^{\prime}, r^{\prime \prime}\right)$, где

$$
r^{\prime}:=|\varsigma+1|=\left|\frac{3}{2}+i \frac{1}{2 \sqrt{3}}\right|=\sqrt{\frac{7}{3}}, r^{\prime \prime}:=2 \cos \frac{\pi}{6}=\sqrt{3} .
$$

Ясно, что $1<r^{\prime}<r^{\prime \prime}$. Стало быть, сопряженные диаграммы $\bar{D}_{A}, \bar{D}_{B}$ функций $A\left(z^{3}\right)$ и $B\left(z^{3}\right)$ лежат в круговой области $\Omega_{r}:=\{z:|z|<r\}$. Значит,

$$
A\left(z^{3}\right), B\left(z^{3}\right) \in P\left(\Omega_{r}\right) .
$$

Сопряженная диаграмма $\bar{D}_{A B}$ произведения $A\left(z^{3}\right) B\left(z^{3}\right)$ совпадает с правильным шестиугольником. Вершины этого шестиугольника

$$
\omega_{6}^{0}+\omega_{6}^{1}, \quad \omega_{6}^{1}+\omega_{6}^{2}, \quad \omega_{6}^{2}+\omega_{6}^{3}, \quad \omega_{6}^{3}+\omega_{6}^{4}, \quad \omega_{6}^{4}+\omega_{6}^{5}, \quad \omega_{6}^{5}+\omega_{6}^{6}
$$

лежат вне области $\Omega_{r}$. Действительно, для любых $k \in\{0, \ldots, 5\}$ имеем

$$
\begin{aligned}
\left|\omega_{6}^{k}+\omega_{6}^{k+1}\right|^{2}=\left(\cos \frac{\pi k}{3}+\right. & \left.\cos \frac{\pi(k+1)}{3}\right)^{2}+\left(\sin \frac{\pi k}{3}+\sin \frac{\pi(k+1)}{3}\right)^{2}= \\
& =2+2\left(\cos \frac{\pi k}{3} \cos \frac{\pi(k+1)}{3}+\sin \frac{\pi k}{3} \sin \frac{\pi(k+1)}{3}\right)=2+2 \cos \frac{\pi}{3}=3
\end{aligned}
$$

При этом по свойствам квазиполиномов вдоль лучей

$$
\operatorname{Arg} z=\operatorname{Arg}\left(\omega_{6}^{k}+\omega_{6}^{k+1}\right), \quad k \in\{0, \ldots, 5\}
$$

выполняются асимптотические оценки

$$
A\left(z^{3}\right) B\left(z^{3}\right)>\exp \{\sqrt{3}|z|-o(1)|z|\}, \quad|z| \rightarrow+\infty .
$$

Положим

$$
a\left(z^{3}\right):=\sum_{k=0}^{5} \exp \left\{\omega_{6}^{k} \varsigma z\right\}, \quad \varphi(z):=A\left(z^{3}\right)+z^{n_{s+1}-n_{s}} B\left(z^{3}\right)
$$

и рассмотрим целую функцию

$$
F(z):=z^{n_{s+1}} a\left(z^{3}\right) \varphi(z)=z^{n_{s+1}} a\left(z^{3}\right) A\left(z^{3}\right)+z^{2 n_{s+1}-n_{s}} a\left(z^{3}\right) B\left(z^{3}\right) .
$$


Сопряженная диаграмма функции $a\left(z^{3}\right)$ совпадает с шестиугольником $\bar{D}_{A} \cap \bar{D}_{B}$, а сопряженная диаграмма функции $\varphi(z)$ совпадает с выпуклой оболочкой множества $\bar{D}_{A} \cup \bar{D}_{B}$, значит, сопряженная диаграмма $\bar{D}_{F}$ функции $F(z)$ совпадает с выпуклой оболочкой множества

$$
\left(\bar{D}_{A} \cap \bar{D}_{B}+\bar{D}_{A}\right) \cup\left(\bar{D}_{A} \cap \bar{D}_{B}+\bar{D}_{B}\right) .
$$

Из геометрических соображений вытекает, что множество $\bar{D}_{F}$ лежит в круге $|z| \leqslant r^{\prime}:=|\varsigma+1|$. Значит, целая функция $F(z)$ тоже принадлежит пространству $P\left(\Omega_{r}\right)$ и, следовательно, 2-функция

$$
\boldsymbol{a}(z):= \begin{cases}(0, a(z)), & \text { если } s=1, \\ (z a(z), 0), & \text { если } s=2,\end{cases}
$$

принадлежит пространству $\boldsymbol{O}_{\varphi}:=\boldsymbol{O}_{\varphi}\left(\Omega_{r}\right)$.

Предположим, что произвольное решение $f \in O\left(\Omega_{r}\right)$ однородного уравнения 3 можно аппроксимировать элементарными решениями этого уравнения в топологии пространства $O\left(\Omega_{r}\right)$. Тогда в силу предложения 1 и теоремы 22 -полиномы плотны в пространстве $\boldsymbol{O}_{\varphi}$. Значит, существует обобщенная последовательность $\boldsymbol{p}_{\sigma}(z):=\left(p_{\sigma, 1}(z), p_{\sigma, 2}(z)\right) 2$-полиномов, сходящаяся к 2-функции $\boldsymbol{a}(z)$ в топологии пространства $\boldsymbol{O}_{\varphi}$. Значит, обобщенная последовательность

$$
\Phi_{\sigma}(z):=p_{\sigma}(z)\left(A\left(z^{3}\right)+z^{n_{s+1}-n_{s}} B\left(z^{3}\right)\right)=\left(z^{n_{1}} p_{\sigma, 1}\left(z^{3}\right)+z^{n_{2}} p_{\sigma, 2}\left(z^{3}\right)\right)\left(A\left(z^{3}\right)+z^{n_{s+1}-n_{s}} B\left(z^{3}\right)\right)
$$

сходится к функции $F(z)$ в топологии пространства $P\left(\Omega_{r}\right)$. При этом

$$
p_{\sigma}(z):=z^{n_{1}} p_{\sigma, 1}\left(z^{3}\right)+z^{n_{2}} p_{\sigma, 2}\left(z^{3}\right) \rightarrow z^{n_{s+1}} a\left(z^{3}\right)
$$

равномерно на компактах. Если $s=1$, то в силу предложения 2

$$
p_{\sigma, 2}\left(z^{3}\right)=\frac{1}{3 z^{n_{2}}} \sum_{k=0}^{2} \omega_{3}^{-n_{2} k} p_{\sigma}\left(\omega_{3}^{k} z\right) \rightarrow a\left(z^{3}\right)
$$

равномерно на компактах. Если $s=2$, то

$$
p_{\sigma, 1}\left(z^{3}\right)=\frac{1}{3} \sum_{k=0}^{2} p_{\sigma}\left(\omega_{3}^{k} z\right) \rightarrow z^{3} a\left(z^{3}\right)
$$

равномерно на компактах. Ниже мы покажем, что соотношения (7) и (8) невозможны.

Воспользуемся представлением

$$
\Phi_{\sigma}(z)=z^{k_{s}} P_{\sigma}\left(z^{3}\right) B\left(z^{3}\right)+C_{\sigma}(z),
$$

где

$$
\begin{gathered}
k_{s}:=\left\{\begin{array}{ll}
2 n_{s+1}-n_{s}, & \text { если } s=1, \\
2 n_{s+1}-n_{s}-3, & \text { если } s=2,
\end{array} \quad P_{\sigma}\left(z^{3}\right):= \begin{cases}p_{\sigma, 2}\left(z^{3}\right), & \text { если } s=1, \\
p_{\sigma, 0}\left(z^{3}\right), & \text { если } s=2,\end{cases} \right. \\
C_{\sigma}(z)=\sum_{n \in n_{A}} z^{n} p_{\sigma, n}\left(z^{3}\right) A\left(z^{3}\right)+z^{n_{s}} p_{\sigma, n_{s}}\left(z^{3}\right) B\left(z^{3}\right)
\end{gathered}
$$

Выберем $r_{1} \in\left(r, r^{\prime \prime}\right)$ и рассмотрим множество

$$
V:=\left\{\psi \in P\left(\Omega_{r}\right):|\psi(z)| \leqslant \exp r_{1}|z|\right\} .
$$

Это множество является окрестностью нуля в $P\left(\Omega_{r}\right)$ (см. [1, теорема 3.3]). При некотором $M \geqslant 1$ все $\Phi_{\sigma}(z)$ принадлежат $M V$. Тогда для всех $z \in \mathbb{C}$ и всех $\sigma \in \sum$ будет выполняться неравенство

$$
\left|\Phi_{\sigma}(z)\right| \leqslant M \exp r_{1}|z| .
$$

Выберем $r_{2} \in\left(r_{1}, r^{\prime \prime}\right)$ и рассмотрим целую 6-симметричную функцию

$$
D\left(z^{3}\right):=\sum_{k=0}^{5} \exp \left[\left(r_{2}-r_{1}\right) \gamma \omega_{6}^{k} z\right] .
$$


В силу (6) при некоторых $r_{3} \in\left(0, r^{\prime \prime}-r_{2}\right), M^{\prime}>0$ на границе $\partial G$ множества $G$ имеет место равномерная по $\sigma \in \sum$ оценка

$$
\left|\frac{D\left(z^{3}\right) \Phi_{\sigma}(z)}{A\left(z^{3}\right) B\left(z^{3}\right)}\right| \leqslant M^{\prime} \exp \left[-r_{3}|z|\right] .
$$

Отступим от границы множества $G$ и рассмотрим множество $G_{\theta^{\prime}}:=\left\{z:|\arg z|<\theta^{\prime},|z|>1\right\} \subseteq G$, где $\theta^{\prime} \in(0, \theta)$. Выберем произвольную точку $z \in G_{\theta^{\prime}}$ и проинтегрируем функцию

$$
\frac{\Phi_{\sigma}(h) D\left(h^{3}\right) h^{5-k_{s}}}{A\left(h^{3}\right) B\left(h^{3}\right)\left(h^{3}-z^{3}\right)}
$$

по положительно ориентированной границе $(\partial G)_{+}$множества $G$. В силу $(5)$ при $s=1$ выполняются неравенства $k_{s}:=2 n_{2}-n_{1}<n_{3} \leqslant 4$, значит, $k_{s} \leqslant 3$. При $s=2$ имеем $k_{s}:=n_{3}-n_{2}=$ $3-\left(n_{2}-n_{1}\right)<3$. Следовательно, интегрируемая функция аналитична в граничных точках множества $G$. Используя представление (9), получим

$$
I_{\sigma}(z)=I_{\sigma, 1}(z)+I_{\sigma, 2}(z),
$$

где

$$
\begin{gathered}
I_{\sigma}(z):=\int_{(\partial G)_{+}} \frac{\Phi_{\sigma}(h) D\left(h^{3}\right) h^{5-k_{s}}}{A\left(h^{3}\right) B\left(h^{3}\right)\left(h^{3}-z^{3}\right)} d h, \quad I_{\sigma, 1}(z):=\int_{(\partial G)_{+}} \frac{P_{\sigma}\left(h^{3}\right) D\left(h^{3}\right) h^{5}}{A\left(h^{3}\right)\left(h^{3}-z^{3}\right)} d h, \\
I_{\sigma, 2}(z):=\int_{(\partial G)_{+}} \frac{C_{\sigma}(h) D\left(h^{3}\right) h^{5-k_{s}}}{A\left(h^{3}\right) B\left(h^{3}\right)\left(h^{3}-z^{3}\right)} d h .
\end{gathered}
$$

Из оценки (10) вытекает равномерная по $\sigma \in \sum$ по $z \in \partial G$ оценка

$$
\left|I_{\sigma}(z)\right| \leqslant \text { const. }
$$

Поскольку все нули целой функции $A\left(z^{3}\right)$ лежат в $G^{\prime}=\mathbb{C} \backslash \bar{G}$, то

$$
I_{\sigma, 1}(z)=\left.\frac{P_{\sigma}\left(z^{3}\right) D\left(z^{3}\right) z^{3}}{A\left(z^{3}\right)} \sum_{k=0}^{2}\left(\frac{h^{2}}{3 h^{2}}\right)\right|_{h=\omega_{3}^{k} z}=\frac{P_{\sigma}\left(z^{3}\right) D\left(z^{3}\right) z^{3}}{A\left(z^{3}\right)} .
$$

Покажем, что интеграл $I_{\sigma, 2}(z)$ равен нулю. Действительно,

$$
I_{\sigma, 2}(z)=\sum_{n \in n_{A}} I_{\sigma, n}^{\prime}(z)+I_{\sigma}^{\prime \prime}(z)
$$

где

$$
\begin{gathered}
I_{\sigma, n}^{\prime}(z)=\int_{(\partial G)_{+}} \frac{p_{\sigma, n}\left(h^{3}\right) D\left(h^{3}\right) h^{5+n-k_{s}}}{B\left(h^{3}\right)\left(h^{3}-z^{3}\right)} d h=-\int_{\left(\partial G^{\prime}\right)_{+}} \frac{p_{\sigma, n}\left(h^{3}\right) D\left(h^{3}\right) h^{5+n-k_{s}}}{B\left(h^{3}\right)\left(h^{3}-z^{3}\right)} d h, \\
I_{\sigma}^{\prime \prime}(z)=\int_{(\partial G)_{+}} \frac{p_{\sigma, n_{s}}\left(h^{3}\right) D\left(h^{3}\right) h^{5+n_{s}-k_{s}}}{A\left(h^{3}\right)\left(h^{3}-z^{3}\right)} d h .
\end{gathered}
$$

Поскольку все нули целой функции $B\left(h^{3}\right)$ лежат в $G$ и $z \in G_{\theta^{\prime}} \subseteq G$, то $I_{\sigma, n}^{\prime}(z)=0$ для любого $n \in n_{A}$. При этом все нули целой функции $A\left(z^{3}\right)$ лежат в $G^{\prime}=\mathbb{C} \backslash \bar{G}$, значит,

$$
\begin{aligned}
& I_{\sigma}^{\prime \prime}(z)=\sum_{k=0}^{2} \int_{\left(\partial G_{k}\right)_{+}} \frac{p_{\sigma, n_{s}}\left(h^{3}\right) D\left(h^{3}\right) h^{5+n_{s}-k_{s}}}{A\left(h^{3}\right)\left(h^{3}-z^{3}\right)} d h= \\
& \quad=\left.\frac{p_{\sigma, n_{s}}\left(z^{3}\right) D\left(z^{3}\right)}{A\left(z^{3}\right)} \sum_{k=0}^{2}\left(\frac{h^{5+n_{s}-k_{s}}}{3 h^{2}}\right)\right|_{h=\omega_{3}^{k} z}=\frac{p_{\sigma, n_{s}}\left(z^{3}\right) D\left(z^{3}\right) z^{3+n_{s}-k_{s}}}{3 A\left(z^{3}\right)} \sum_{k=0}^{2} \omega_{3}^{k\left(n_{s}-k_{s}\right)}=0,
\end{aligned}
$$


так как целое число $l_{s}:=n_{s}-k_{s}$ не делится на 3 для любого $s \in\{1 ; 2\}$. Действительно, если $s=1$, то в силу (4) и (5) имеем $-3=n_{1}-n_{3}<l_{s}=-2 n_{2}<0$. Если $s=2$, то $-6=n_{2}-n_{4}-3<l_{s}=$ $2 n_{2}-2 n_{1}-3<-3$. Далее, в силу (11)

$$
\left|z^{3} P_{\sigma}\left(z^{3}\right) D\left(z^{3}\right)\right| \leqslant \text { const }\left|A\left(z^{3}\right)\right|
$$

для любого $z \in G_{\theta^{\prime}}$ и любого $\sigma \in \Sigma$. Значит, в силу (7) и (8) для предельной функции имеем:

$$
\begin{aligned}
& \left|z^{3} a\left(z^{3}\right) D\left(h^{3}\right)\right| \leqslant \text { const }\left|A\left(z^{3}\right)\right|, \quad z \in G_{\theta^{\prime}}, \quad s=1 ; \\
& \left|z^{6} a\left(z^{3}\right) D\left(h^{3}\right)\right| \leqslant \text { const }\left|A\left(z^{3}\right)\right|, \quad z \in G_{\theta^{\prime}}, \quad s=2 .
\end{aligned}
$$

Но эти неравенства не могут выполняться для всех $z \in G_{\theta^{\prime}}$, если $\theta$ и $\theta^{\prime}$ выбраны достаточно близко к $\pi / 3$, так как рост функций $z^{3} a\left(z^{3}\right) D\left(z^{3}\right)$ и $z^{6} a\left(z^{3}\right) D\left(z^{3}\right)$ на лучах $|\arg z|=\pi / 3$ больше роста функции $A\left(z^{3}\right)$. Необходимость доказана.

\section{СПИСОК ЛИТЕРАТУРЫ}

1. Красичков-Терновский И. Ф. Инвариантные подпространства аналитических функций. І. Спектральный синтез на выпуклых областях // Мат. сб. - 1972. - 87 (129), № 4. - С. 459-489.

2. Красичков-Терновский И. Ф. Инвариантные подпространства аналитических функций. II. Спектральный синтез на выпуклых областях// Мат. сб. - 1972. - 88, № 1. - С. 3-30.

3. Леонтъев А. Ф. Ряды экспонент. - М.: Наука, 1976.

4. Шишкин А. Б. Спектральный синтез для оператора, порождаемого умножением на степень независимой переменной// Мат. сб. - 1991. - 182, № 6. - С. 828-848.

5. Шишкин А. Б. Проективное и инъективное описания в комплексной области. Двойственность// Изв. Саратов. ун-та. Нов. сер. Сер. Мат. Мех. Информ. - 2014. - 14, № 1. - С. 47-65.

6. Шишкин $A$. Б. Экспоненциальный синтез в ядре оператора симметричной свертки// Зап. науч. семин. ПОМИ. - 2016. - 447. - С. 129-170.

Татаркин Александр Александрович

Кубанский государственный университет, Краснодар

E-mail: tiamatory@gmail.com

Шишкин Андрей Борисович

Кубанский государственный университет, Краснодар

E-mail: shishkin-home@mail.ru 